\section{AB1018 18 TREATMENT RESPONSE OF HYDRONEPHROSIS ASSOCIATED WITH IDIOPATHIC RETROPERITONEAL FIBROSIS, FOCUSING ON RATIO OF IGG4/IGG3 SERUM CONCENTRATION}

S. J. Choi ${ }^{1}$, S. H. Nam ${ }^{1}$, J. S. Lee ${ }^{1}$, W. J. Seo ${ }^{2}$, J. S. Oh ${ }^{3}$, S. Hong ${ }^{1}$, C. K. Lee ${ }^{1}$, B. Yoo ${ }^{1}$, Y. G. Kim ${ }^{1.1}$ University of Ulsan College of Medicine, Asan Medical Center, Division of Rheumatology, Department of Internal Medicine, Seoul, Korea, Rep. of (South Korea); ${ }^{2}$ Seoul Veterans Hospital, Division of Rheumatology, Department of Internal Medicine, Seoul, Korea, Rep. of (South Korea); ${ }^{3}$ Asan Medical Center, Department of Biomedical Informatics, Seoul, Korea, Rep. of (South Korea)

Background: Hydronephrosis, a common complication of idiopathic retroperitoneal fibrosis (iRPF), may lead to poor renal outcomes unless it is resolved. Pathological confirmation can help to identify the aetiology of the disease and determine the treatment strategy. But, in most cases, it is difficult to obtain sufficient tissue due to the location of fibrosis. In a recent study, parts of iRPF are correlated with IgG4-related disease characterised by elevated serum IgG4 levels $(>135 \mathrm{mg} / \mathrm{dL})$. Normal serum IgG3 level $(21-176 \mathrm{mg} / \mathrm{dL})$ has been known to be higher than normal serum IgG4 level (4-86 mg/dL). The reverse IgG4/lgG3 ratio has been suggested to be an IgG4-related disease component that distinguishes it from primary sclerosing cholangitis [1]. However, the ratio of IgG3 and IgG4 may be reversed in iRPF patients with hydronephrosis.

Objectives: We aimed to investigate the ratio of IgG subclasses as a predictive factor associated with treatment response of hydronephrosis in patients with iRPF. Methods: We retrospectively recruited 19 iRPF patients with hydronephrosis who evaluated serum IgG subclasses in a tertiary hospital between 2004 and 2019. Hydronephrosis was evaluated on the basis of imaging findings. Medications and clinical and laboratory findings, including IgG subclasses, were reviewed following the diagnosis of hydronephrosis. Hydronephrosis improvement on subsequent images was evaluated to assess treatment response. Categorised data were compared using chi-square or Fisher's exact test. Continuous variables were compared using Mann-Whitney $U$ test.

Results: At baseline, median serum IgG3 and IgG4 levels were 64 (IQR 3782) $\mathrm{mg} / \mathrm{dL}$ and 71 (IQR 40-171) $\mathrm{mg} / \mathrm{dL}$. Five patients had serum IgG4 levels > $135 \mathrm{mg} / \mathrm{dL}$ and 11 patients had the reverse serum level of IgG4/lgG3. On subsequent images (median follow-up at 3.2 [IQR 1.7-4.0] months), 11 patients showed hydronephrosis improvement. The proportions of positive ratio of serum $\operatorname{lgG} 4 / \operatorname{lgG} 3(81.8 \%$ vs. $25 \%, p=0.024)$, periaortic involvement $(81.8 \%$ vs. $25 \%$, $p=0.024)$ and high-dose glucocorticoid treatment $(45.5 \%$ vs. $0 \%, p=0.045)$ were significantly higher in patients with improvement than in those without improvement (Table 1). Interestingly, even in cases with normal serum IgG4 levels, patients with improvement showed a higher serum IgG4/lgG3 ratio than in those without improvement (median 1.5 vs. $0.7, p=0.038$ ).

Table 1. Clinical characteristics and treatment according to the shortterm outcome of hydronephrosis

\begin{tabular}{lccc}
\hline & $\begin{array}{c}\text { Improvement } \\
(\mathrm{n}=11)\end{array}$ & $\begin{array}{c}\text { No improvement } \\
(\mathrm{n}=8)\end{array}$ & p-value \\
\hline Time to subsequent imaging (months) $^{\mathrm{a}}$ & $2.2(1.3-4.2)$ & $3.2(2.0-3.8)$ & 0.778 \\
Males (n, \%) & $8(72.7 \%)$ & $7(87.5 \%)$ & 0.603 \\
Periaortic involvement (n, \%) & $9(81.8 \%)$ & $2(25 \%)$ & 0.024 \\
Impaired renal function (n, \%) $^{\mathrm{b}}$ & $5(45.5 \%)$ & $2(25 \%)$ & 0.633 \\
Serum IgG4 (mg/dL) & $114(59-172)$ & $43(35-109)$ & 0.152 \\
Elevated serum IgG4 (n, \%) $^{\mathrm{a}}$ & $4(36.4 \%)$ & $1(12.5 \%)$ & 0.338 \\
Serum IgG4/lgG3 ratio & $2.1(1.2-4.9)$ & $0.8(0.4-1.0)$ & 0.041 \\
Positive ratio of serum IgG4/IgG3 (n, \%) & $9(81.8 \%)$ & $2(25 \%)$ & 0.024 \\
Medical treatment (n, \%) & $7(63.6 \%)$ & $1(12.5 \%)$ & 0.059 \\
High-dose glucocorticoid treatment (n, \%) & $5(45.5 \%)$ & $0(0 \%)$ & 0.045 \\
Surgical intervention (n, \%) & $8(72.7 \%)$ & $4(50 \%)$ & 0.377 \\
\hline
\end{tabular}

${ }^{\text {a }}$ Values are median and interquartile range $\left(25^{\text {th }}-75^{\text {th }}\right.$ percentile $)$

${ }^{\mathrm{b}}$ Defined as serum creatinine level $>1.24 \mathrm{mg} / \mathrm{dL}$

Conclusion: The reverse ratio of serum IgG4/lgG3 was associated with hydronephrosis treatment response, thus suggesting favourable responses to highdose corticosteroid.

References:

[1] Boonstra K, Culver EL, de Buy Wenniger LM, et al. Serum immunoglobulin G4 and immunoglobulin G1 for distinguishing immunoglobulin G4-associated cholangitis from primary sclerosing cholangitis. Hepatology 2014;59:1954-63.

Disclosure of Interests: None declared

DOI: 10.1136/annrheumdis-2020-eular.4159

\begin{tabular}{|l|l}
\hline AB1019 & THERAPEUTIC PROFILE OF PATIENTS WITH VOGT \\
KOYANAGI HARADA DISEASE FOLLOWED AT THE \\
HOSPITAL DE CLÍNICAS. SAN LORENZO PARAGUAY
\end{tabular}

N. Colman ${ }^{1}$, J. Losanto ${ }^{1}$, S. Riquelme ${ }^{1}$, M. Melo ${ }^{1}$ on behalf of Autoimmune Eye Diseases Unit. Hospital de Clínicas. PY. ${ }^{1}$ Hospital de Clínicas, San Lorenzo, Paraguay

Background: Vogt-Koyanagi-Harada Disease (VKHD) is an autoimmune eye disease that causes chronic vision complications in young people. The treatment is based on corticosteroids and immunosuppressants, to which biological drugs are currently added. The visual prognosis depends on the stage in which it is diagnosed and the effective and timely treatment. ${ }^{1,2}$

Objectives: Describe the evolution and the therapeutic profile of patients with VKHD who go to the Rheumatology Department of the Hospital de Clínicas. San Lorenzo Paraguay until November 2019

Methods: Descriptive cross-sectional study conducted at the Hospital de Clínicas, San Lorenzo Paraguay, where clinical histories of patients with this disease and who have regular follow-up were reviewed.

Results: 21 patients were included, 17 (81\%) women, average age of 35 years. The diagnosis and treatment were made $15(71 \%)$ in acute phase, $4(19 \%)$ convalescent phase and $2(10 \%)$ in chronic phase.

Corticosteroids: All received corticosteroids at diagnosis. Methylprednisolone IV $1 \mathrm{~g} /$ day for 3 consecutive days in 8 (44\%) cases and for 5 days in 10 (55.5\%) cases. The first month everyone received prednisone orally at $1 \mathrm{mg} / \mathrm{kg} / \mathrm{d}$ on average $(60-75 \mathrm{mg} / \mathrm{d})$, at 3 months the average dose of prednisone was $37 \mathrm{mg} /$ $\mathrm{d}$, at 6 months of $23 \mathrm{mg} / \mathrm{d}$ and at 12 months $10 \mathrm{mg} / \mathrm{d}$ months.

Immunosuppressive treatment: They received 20 (95\%) patients. Induction with monthly intravenous cyclophosphamide was performed in $8(40 \%)$ cases and with Azathioprine in $12(60 \%)$ cases. Of the patients treated with Azathioprine initially, in 5 (23\%) patients it was necessary to switch to Cyclophosphamide IV due to failure or intolerance. The maintenance treatment was azathioprine in 13 $(62 \%)$ of the patients, MTX in $4(19 \%)$ and the association of both drugs was indicated in $3(14 \%)$ patients. One case was refractory and received infliximab and subsequently Rituximab, which resulted in remission.

The best final Visual Acuity (AV) was recorded with the Snellen chart in 40 eyes (20 patients), 27 (67.5\%) patients with good vision (20/20 to $20 / 50), 8(20 \%)$ patients with decreased VA (20 / 50-20 / 200), and 5 (12.5\%) with legal blindness $(<20 / 200)$ and of these only 3 patients had unilateral involvement and one patient with bilateral blindness. Sequelae / complications: 6 (28.5\%) patients presented cataracts and finally $3(14 \%)$ glaucoma, 5 (24\%) anterior / posterior synechiae. Conclusion: The majority of patients in this series presented a good vision when the inflammatory process was resolved (AV 20 / 20-20 / 50), this probably related to early diagnosis and initial aggressive treatment with intravenous corticosteroid pulses and its association with immunosuppressants of entry as mentioned in the literature. The worst vision, (visual acuity $<20 / 200$ ) occurred in 5 of 40 eyes, corresponding to 4 patients, two of them diagnosed and treated in the chronic stage. Timely treatment improves visual prognosis.

References:

[1] Lodhi SAK, Lokabhi Reddy JM, Peram V. Clinical spectrum and management options in Vogt-Koyanagi-Harada disease. Clin Ophthalmol. 2017;11:1399-406.

[2] Concha-del Río LE, Gómez L, Arellanes-García L. Corticotherapy vs. Corticotherapy Plus Immunosuppressive Therapy in Acute Vogt-Koyanagi-Harada Disease. Arch Soc Esp Oftalmol [Internet]. 2018;93(5):225-30. Available from: https://doi.org/10.1016/j.oftal.2017.09.010

Acknowledgments: Department of Rheumatology

Department of Ophthalmology

Disclosure of Interests: None declared

DOI: 10.1136/annrheumdis-2020-eular.792

\section{AB1020 \\ APPLICATION OF THE 2019 ACR/EULAR CLASSIFICATION CRITERIA CONTRIBUTES TO THE DIAGNOSIS OF IGG4-RELATED DISEASE}

L. F. Chen ${ }^{1}$, J. J. Liang ${ }^{1}$, Z. M. Ouyang ${ }^{1}$, J. D. MA ${ }^{1}$, D. H. Zheng ${ }^{1}$, L. Dai ${ }^{1} .{ }^{1}$ Sun Yat-Sen Memorial Hospital, Sun Yat-Sen University, Rheumatology, Guangzhou, China

Background: IgG4-related disease (IgG4-RD) is a rare and systemic disease characterized by swelling or masses in the involved organs, elevated serum IgG4 marked IgG4+ plasma cells infiltration and fibrosis. However, lacking of pathological data limited the definite diagnosis of IgG4-RD. As results of high-quality clinical and epidemiological investigations collected, new classification criteria for 